\title{
Choices on selective clinical data sharing by people with Parkinson's disease
}

\author{
Leah R Mursaleen ${ }^{1,2}$ \\ Jon A Stamford ${ }^{1,2}$ \\ Peter Schmidt ${ }^{3}$ \\ John M Dean ${ }^{4}$ \\ Richard Windle ${ }^{2}$ \\ David A Jones ${ }^{2}$ \\ Helen Matthews ${ }^{1,2}$ \\ 'The Cure Parkinson's Trust, London, \\ UK; ${ }^{2}$ Parkinson's Movement, London, \\ UK; ${ }^{3}$ National Parkinson Foundation, \\ Parkinson's Foundation, Miami, FL, \\ USA; ${ }^{4}$ Davis Phinney Foundation, \\ Boulder, CO, USA
}

This article was published in the following Dove Press journal: Journal of Parkinsonism and Restless Legs Syndrome 26 June 2017

Number of times this article has been viewed

Background: Clinical data sharing and ownership are key issues in modern digital data acquisition. Data sharing is subject to influence by a range of stakeholders. Of these, patient attitudes are pivotal.

Objectives: The objective of this report was to characterize attitudes to clinical data sharing among people with Parkinson's disease (PD).

Methods: A recent survey, conducted by the Parkinson's Movement (2016) highlighted patient concerns over data sharing. This formed the basis for discussion by two focus groups at the Rallying to the Challenge meeting at the Van Andel Research Institute in September 2016.

Results: The focus groups examined issues related to the appropriateness of data sharing for different categories of data and highlighted both the value and concerns regarding data sharing. Conclusion: At the conclusion of the session, it was proposed that a "data charter" be developed to reflect the thinking of people with PD on best practices in data acquisition, ownership, and sharing. Keywords: Parkinson's disease, patient choice, data sharing, data acquisition, data ownership, best practices, data charter

\section{Introduction}

Many organizations collect personal health-related information, compile and organize it into anonymized data, and make it available to researchers. ${ }^{1}$ Such data and its sources include, but are not limited to, clinical and public health records, fitness trackers, health apps, biometric sensors, websites, and social media. In the vast majority of cases, the data is in a digital format. Digitally generated and stored data has greater longevity than analog data, being potentially infinite in lifespan and uncapped in its capacity for reuse and re-analysis. ${ }^{1}$ Collection of this information may be useful in the identification of patterns in diseases, differences in populations, and may ultimately help to identify unmet needs and shape research questions. ${ }^{2}$ Unsurprisingly, therefore, this approach has the potential to cause significant impact in numerous disease areas, including Parkinson's disease (PD).

Collecting information on personal experiences from people with $\mathrm{PD}(\mathrm{PwP})$ using any of the above methodologies may help to provide insights about their personal experience with PD. For example, information about symptom frequency and its severity has the potential to identify clinically meaningful patterns in an individual's disease. This would be particularly relevant for PwP as some PwP acknowledge not communicating the full range of their symptoms to their healthcare providers and others. ${ }^{3}$ Collecting personal experiences of PwP can also shape scientific and clinical expertise. Achieving

Correspondence: Leah R Mursaleen The Cure Parkinson's Trust, 120 Baker Street, London WIU 6TU, UK Tel +442074873892

Email leah@cureparkinsons.org.uk 
these potential benefits necessitates careful and appropriate data sharing with the scientific community.

In the case of research on PD, sharing of patient-level digital data has been limited by issues of ownership restricting other research stakeholders' access to the data. ${ }^{4}$ This is particularly relevant with respect to mobile applications and wearable devices, where company mergers and acquisitions can alter the accepted data management policies. There are thus numerous questions that need to be answered surrounding data sharing and data collection. Further to this, there is clear discordance between symptoms that are currently measured and what PwP feel are the symptoms most useful to measure in terms of quality of life. ${ }^{3}$

\section{Method}

A 37 question online survey was carried out by the Parkinson's Movement (www.parkinsonsmovement.com), an international patient-driven action group created by The Cure Parkinson's Trust (www.cureparkinsons.org.uk), a UK research charity. The survey (Supplementary material 1) focused upon understanding what information PwP are willing to share and how that information can influence decision making in healthcare and research. ${ }^{5}$ Completion of the survey was deemed to be consent from the participants.

The key findings of this survey are presented in Supplementary material 2 and were discussed at the 2016 Rallying to the Challenge meeting, which was organized by the Van Andel Research Institute in association with Parkinson's Movement. This meeting was attended by 43 PwP predominantly from the US and was organized specifically to discuss the questions surrounding sharing information and data collection. The highlights of the questionnaire served as a focus of discussion for the advocates, researchers, PwP, and care partners in attendance in an effort to address the issues around data sharing and collection.

Two focus groups were convened from the $43 \mathrm{PwP}$ conference participants, randomly assigned to either group. Each group had two facilitators, one from The Cure Parkinson's Trust and the other from either the Davis Phinney Foundation or the National Parkinson's Foundation. One facilitator prompted discussion while the second captured discussion points and conclusions. The focus groups were asked to address three questions:

\section{Focus group I}

- What data is engaging for people to share?

- What data should be collected?

- What data is needed?

\section{Focus group 2}

- How can we inspire people to provide their information?

- What personal value is provided by sharing data?

- Who should own the shared data?

Each focus group was of 60-minute duration with equal time allocated to each question. On conclusion of the focus group, its findings were disseminated among the conference participants.

\section{Results}

Focus group 1 identified three different types of information (in terms of what should be collected and shared); 1) information that should never be collected, 2) one-off information/ information that should only be collected and shared occasionally, and 3) information that should always be collected and shared (Figure 1).

Examples of information that participants suggested should "never be collected" were any identifying data that could be used to influence third party decisions such as issuance of a driving license, insurance, and employment, as well as other personal information such as sexual preference. It was also highlighted that unique identifiers (rather than individual names) should be created to provide assurance of anonymity. Although some demographic information may always be shared such as age at diagnosis, other demographics may only sometimes be shared if necessary, for example, country of residence, date of diagnosis, employment history, family history, and first/presenting symptoms (Figure 1) depending on the purpose for the data that are being collected.

It was also reported that an individual's PD symptoms (for example, motor function, mood/feelings, psychosis, cognition, anxiety, depression, autonomic, speech, swallowing, pain, dystonia, sleep, and fatigue) and their fluctuations in relation to times when medication is wearing off ("off" times) should always be recorded when collecting data for research purposes. In addition to these, it was argued that treatment (e.g. medication, speech therapy, physiotherapy and occupational therapy, surgical treatments, and alternative therapies/ treatments) and information about appointments with their healthcare team (e.g. when, with whom, frequency, duration, wait-time; satisfaction, questions answered, shared decision making, and health confidence) should always be collected when collecting data for research purposes.

Focus group 2 concluded that PwP are more likely to share their information if there is assured anonymity and transparency about the use of their data. It was argued that, if these conditions were met, more PwP would share their 


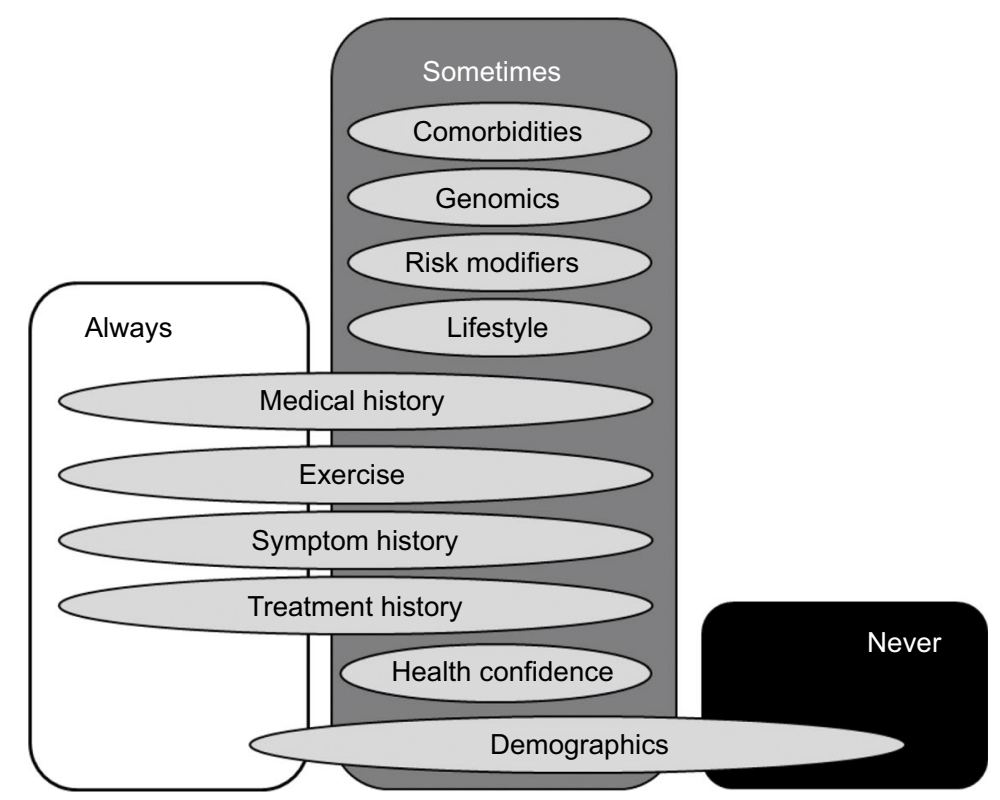

Figure I Examples of data that participants suggested should always be collected, never be collected and should sometimes be collected. Some categories of information such as demographics may overlap between all three groups.

data. This, in turn, might reveal personal and collective insights into the condition. Most participants in the focus group agreed that data being shared by an individual must be owned by that individual. But in any case, it was unanimous that there must be transparency from the outset about how the data is to be used: any changes should be communicated in an on-going dialogue.

\section{Discussion}

Data collection and sharing are issues of significant interest to PwP and the scientific community. However, it is essential that data sharing policies take into consideration the effects of sharing data on participants. ${ }^{6}$ Although, data collection is regulated, different data collection mechanisms exist under different governance regimes. ${ }^{1}$ Depending on the circumstances for which the data is being collected, governance can be a result of law, public health ethics, ethics of clinical care, research ethics, or commercial contracts between individuals and service providers. ${ }^{1}$ That said, many medical apps and devices which perform organized collection of personal data are not subject to government regulation and fall short of data protection standards. ${ }^{7,8}$ It is, therefore, immensely important that PwP define how they want their data to be used. The 2016 Rallying to the Challenge meeting was organized with this in mind.

In the pre-meeting survey, over $75 \%(n=310)$ of the respondents thought both non-motor and motor symptoms should be recorded for research purposes. In concordance, focus group 1 further concluded that an individual's PD symptoms should always be recorded as well as their frequency and severity in relation to "on" and "off" times. Focus group 2 emphasized the importance of patient anonymity and transparency of data usage.

Collectively, these findings from PwP (the primary stakeholder group), should guide best practices for data acquisition and research usage. In many aspects, the discussion by the focus groups highlighted ethical issues around personal data collection and research usage thereof. Currently, few PwP are sharing data for research purposes, ${ }^{5}$ a significant untapped resource. Therefore, it would be useful to produce a PD-specific data charter to outline best practices and highlight appropriate ethical expectations for both PwP sharing their data and groups collecting data.

\section{Acknowledgment}

We thank the Van Andel Research Institute for facilitating the Rallying to the Challenge patient meeting and discussions which form the basis of this paper.

\section{Disclosure}

The authors report no conflicts of interest in this work.

\section{References}

1. O'Doherty KC, Christofides E, Yen J, et al. If you build it, they will come: unintended future uses of organised health data collections. $B M C$ Med Ethics. 2016;17(1):54.

2. Anderson M, Kimberly McCleary K. On the path to a science of patient input. Sci Transl Med. 2016;8(336):336ps11.

3. Mathur S, Mursaleen L, Stamford J, DeWitte S, Robledo I, Isaacs T. Challenges of improving patient-centered care in Parkinson's disease. J Parkinsons Dis. 2017;7(1):163-174. 
4. Dubman S. Call to action: sharing of patient-level data for Parkinson's research and care. Available from: http://www.journalofparkinsonsdisease. com/call-action-sharing-patient-level-data-parkinson $\%$ E2 $\% 80 \% 99$ sresearch-and-care. Accessed November 16, 2016.

5. The Cure Parkinson's Trust. Rallying to the Challenge 2016: Data, Data, Data. Available from: https://www.surveymonkey.co.uk/r/rallying16. Accessed February 08, 2017.
6. Tsai AC, Kohrt BA, Matthews LT, et al. Promises and pitfalls of data sharing in qualitative research. Soc Sci Med. 2016;169:191-198.

7. Cortez NG, Cohen IG, Kesselheim AS. FDA regulation of mobile health technologies. N Engl J Med. 2014;371(4):372-379.

8. Huckvale K, Prieto JT, Tilney M, Benghozi P J, Car J. Unaddressed privacy risks in accredited health and wellness apps: a-cross sectional systematic assessment. BMC Med. 2015;13:214.
Journal of Parkinsonism and Restless Legs Syndrome

\section{Publish your work in this journal}

Journal of Parkinsonism and Restless Legs Syndrome is an online, open access, peer-reviewed journal. The journal publishes review articles, historical reviews, original research articles, case reports, letters to the editor, clinical teaching cases, neuroradiology highlights, neuropathology highlights, neuropsychiatry highlights, autobiographies, conference

Submit your manuscript here: https://www.dovepress.com/journal-of-parkinsonism-and-restless-legs-syndrome-journal

proceedings, abstracts and book reviews. The manuscript management system is completely online and includes a very quick and fair peerreview system, which is all easy to use. Visit http://www.dovepress.com/ testimonials.php to read real quotes from published authors. 